\title{
A BEHAVIOUR-BASED APPROACH TO REACTIVE NAVIGATION FOR AUTONOMOUS ROBOT
}

\author{
D.Y. Yoon ${ }^{* \dagger}$, S. R. Oh ${ }^{\dagger}$, G.T. Park ${ }^{*}$, B. J. You ${ }^{\dagger}$ \\ "Dept. of Electrical Engineering, Korea Univ., \\ ${ }^{\dagger}$ Intelligent System Control Research Center, Korea Institute of Science and \\ Technology(KIST): 39-1, Haweolgog-dong, Seongbuk-gu, Seoul, Korea.
}

\begin{abstract}
This paper proposes a new effective behaviour-based reactive navigation methodology for autonomous mobile robot. Its main contribution is based on newly proposed egocentric 'homeostatic' approach by which behaviours are coordinated while taking advantages of both competitive and cooperative method. In order to accomplish autonomous navigation, direction and velocity are modulated automatically by single real value, satisfaction level' without any central or additional module so that this method becomes simple, consistent, and flexible. The validities of the proposed methodology are shown by graphic simulation and experiments with wheeled mobile robot. Copyright ${ }^{\odot} 2002$ IFAC
\end{abstract}

Keywords: robot navigation, robot programming, mobile robots, behaviour, robotics.

\section{BEHAVIOR-BASED REACTIVE NAVIGATION}

Behaviour-based robotics is one of robot control methodology in which the control commands are made out of coordination of predefined behaviors without any mathematical or symbolic modeling of environments in which the robot acts. Mainly due to these non-model based characteristics, this kind of control methodology is inherently reactive since somewhat burdened and time-consuming process of modeling and reasoning is omitted. Concretely, this methodology qualifies behaviours as the fundamental units of control and each behaviour is implemented in the form of sensing-action pair, in other words, sensory motor meaning reactive action triggered by stimulus. Each behaviour components required for intelligent autonomous navigation are designed a priori before robot running(behaviour decomposition) and these behaviours are selected or rejected to make robot's resultant action command in the face of concrete situation of every sampling time step(behaviour coordination). As the result, the time sequences of these reactive action components play a role of path planning and velocity control simultaneously.

This paper is concerned with these behaviour decomposition and coordination in a different view from predecessors. Many creative and successful behaviour decomposition and coordination schemes were presented (Arkin, 1987; Brooks, 1986; Maes, 1989; Saffiotti, Konolige, and Ruspini, 1995). The behaviour coordination methods can be categorized into two classes(Arkin, 1998): cooperative and competitive method. More than two behaviours are fused to make resultant action of the agent in the former methods(Arkin, 1987) while only one, winner behaviour is selected and it makes the resultant action in the latter method(Brooks, 1986). These two coordination schemes, of course, have their own merits and shortages ${ }^{1}$.

Reactive navigation schemes including these behavior-based approach, however, cannot guarantee safety from collision or undesired cyclic behaviour(Mukerjee, and Mali, 1999; Xu, and Tso, 1999) since each behaviour components can be fused into wrong result due to its improper coordination strategy or inadequately tuned component gains(Arkin, 1987; Xu, and Tso, 1999). From these considerations, a coordination scheme taking advantages of both competitive and cooperative manners are required. At the same time, guaranteeing safety is also necessary. In order to satisfy these requirements, a novel coordination scheme based on so called homeostatic strategy is proposed in this paper.

\footnotetext{
${ }^{1}$ It may say that cooperative ones are more adaptive and competitive ones are more rapid and have expectable results. Various behaviour-based robotic schemes can be shown in (Arkin, 1998) for more comparison.
} 


\section{MISSION VERSUS HOMEOSTASIS OF ROBOTS}

Most of the robot control algorithm treats all behaviours of the robot as a same kind of external (sub)mission or (sub)task for the robot to accomplish but the proposed scheme views the robot's behaviours in different sight. It views the robot's behaviours as one of two different kind of action. One is external mission behaviour as usually viewed and the other is so called 'homeostatic behaviours'. The term 'homeostasis', quoted from biology, was used in somewhat extended meaning from its original strict meaning in order to imply the tendency or driving force to maintain desired state of the robot itself, where the desired states include mainly vitality of the robot such as safety from crash, battery level maintenance and etc. In other words, the homeostatic behaviours are those for fundamental necessary conditions for all another kind of missions to be executable. Let us apply and hence explain this concept through an example of autonomous navigation of a mobile robot as follows: A behaviour set

$$
\begin{aligned}
& \mathbf{B}=\{\text { Move-To-Goal, Avoid-Obstacle, } \\
& \text { Stay-On-Path, Move-Ahead }
\end{aligned}
$$

is assumed to be given for reactive navigation(Arkin, 1987). Here, Move-To-Goal, Stay-On-Path and MoveAhead may be considered as (sub)mission behaviours while Avoid-Obstacle had better be viewed as homeostatic behaviour rather than mission because robot's aim is primarily just to arrive goal but Avoid-Obstacle is not robot's final goal but it can be considered as a kind of necessary condition to achieve the goal. Expanding this example, robot behaviours can be categorized as one of homeostatic behaviours and mission behaviours. Now, the relation of these two kinds of behaviours will be used to coordinate behaviours to make the robot's resultant behaviour, that is, velocity vector in case of mobile robot navigation.

\section{BEHAVIOUR DECOMPOSITION AND COORDINATION ALGORITHM}

\subsection{Behaviour decomposition}

Robot's necessary behaviours for the overall navigation mission are decomposed and classified by two factors of homeostasis and 'behaviour sector'. The 'behaviour sector' is introduced additionally for parallel execution of behaviours which don't share same system resources or which don't touch action objectives of others so that they don't need to be coordinated. On the other hand, only the case of robot's purely reactive navigation is considered in this paper, where the proposed reactive navigation has several features as follows:

-Any global informations except direction to goal are not available during the whole operation.

-Robot does not depend on any tools providing global information such as localization since they inherently have some degree of error.

-Memory may be used unless it records history which depends on global information.

-Available informations are only obtained by robot itself with equipped sensors.

- It depends strongly on sensor ability so the objective of navigation is to obtain best performance within equipped sensor ability of course.

From above, a behaviour set $\mathbf{B}$ can be expressed as follows:

$$
\begin{aligned}
& \mathbf{B}=\bigcup_{i} \mathbf{B}_{i} \\
& \mathbf{B}_{i}=\mathbf{B}_{i H} \cup \mathbf{B}_{i M}, \mathbf{B}_{i H} \cap \mathbf{B}_{i M}=\mathbf{0} \\
& \text { where, } i=0,1, \cdots n \\
& n \text { : number of behaviour sector } \\
& \text { B :whole behaviour set } \\
& \mathbf{B}_{i} \text { : set of } i \text { th behaviour sector } \\
& \mathbf{B}_{i H} \text { : homeostatic subset } \\
& \text { of } i \text { th behaviour sector } \\
& \mathbf{B}_{i M} \text { : mission subset } \\
& \text { of } i \text { th behaviour sector } \\
& \text { 0 : null set }
\end{aligned}
$$

Using frame of (2), a behaviour designer can decompose behaviours for navigation into different set. In this research, navigation is accomplished by only three simple behaviours. The proposed behaviour set is decomposed and classified as (3)

$$
\begin{aligned}
n= & 2, \\
\mathbf{B}= & \{\text { InSafety, ToGoal, InResolution }\} \\
= & \mathbf{B}_{1} \cup \mathbf{B}_{2} \\
\mathbf{B}_{1}= & \{\text { InSafety, ToGoal }\} \\
& =\mathbf{B}_{1 H} \cup \mathbf{B}_{1 M} \\
\mathbf{B}_{1 H}= & \{\text { InSafety }\}, \mathbf{B}_{1 M}=\{\text { ToGoal }\} \\
\mathbf{B}_{2}= & \{\text { InResolution }\} \\
& =\mathbf{B}_{2 H} \cup \mathbf{B}_{2 M} \\
\mathbf{B}_{2 H}= & \{\text { InResolution }\}, \mathbf{B}_{2 M}=\mathbf{0}
\end{aligned}
$$

In (3), the behaviour InSafety plays a role of collision avoidance by inhibiting the robot from going danger direction, ToGoal lets the robot move to goal direction and InResolution controls the robot's velocity in harmony with clutter of the environment. This classification of necessary behaviours, together with each role, is illustrated conceptually as Fig. 1. 
Implementation features of each behaviour are described in next section.

\begin{tabular}{l|lc:l}
$\begin{array}{l}\text { homeostatic } \\
\text { subsets }\end{array}$ & $\begin{array}{l}\text { mission } \\
\text { subset }\end{array}$ & $\begin{array}{l}\text { behavior } \\
\text { sectors }\end{array}$ & $\begin{array}{l}\text { physical } \\
\text { role }\end{array}$ \\
\cline { 1 - 1 } $\begin{array}{l}\mathbf{B}_{1 H} \\
=\{\text { InSafety }\}\end{array}$ & $\begin{array}{l}\mathbf{B}_{1 M} \\
=\{\text { ToGoal }\}\end{array}$ & $\mathbf{B}_{1}$ & $\begin{array}{l}\text { to give } \\
\text { the } \\
\text { robot }\end{array}$ \\
\hline $\begin{array}{l}\mathbf{B}_{2 H} \\
=\{\text { InResolution }\}\end{array}$ & $\begin{array}{l}\mathbf{B}_{2 M} \\
=\mathbf{0}\end{array}$ & $\mathbf{B}_{2}$ & $\begin{array}{l}\text { to give } \\
\text { the } \\
\text { robot }\end{array}$ \\
\hline
\end{tabular}

Fig. 1. Decomposition and classification of behaviours for reactive navigation.

\subsection{Implementation of behaviours}

Each decomposed behaviour has two elements. One is its output value corresponding to robot's action and the other is the parameter, here named 'satisfaction level', which will be used for coordination among behaviours.

The barometer of coordination, 'satisfaction level'; The parameter is introduced and implemented in each behaviour in order to compare significance of each behaviour. In addition, it is named as 'satisfaction level' to fit the term more legible to its usage in our scheme. There have been so many works that use explicitly or implicitly this concept of parameter for coordination(Arkin, 1987; Maes, 1989; Saffiotti, Konolige, and Ruspini, 1995). In this research, a major caution about using this concept is emphasized explicitly although all previous works did implicitly:

"Satisfaction level of each behaviour should be made to be 'comparable value at a fair valuation' that is, a specific value of satisfaction level of one behaviour should have equal significance in other behaviours also".

This is a necessary condition for behaviour coordination. Under this condition, it is worth of comparing significance of behaviours in competition. Satisfaction levels is designed concretely by the means as follows:

-Satisfaction levels are decided based on the current sensor data corresponding to its behaviour.

-All Satisfaction levels are real values and linearly scaled into $[0,1]$ regardless of sensor data range.

The first strategy is for reactivity and the second is for comparison and it is assumed that same normalized sensor value has same significance that is, same satisfaction level. Satis faction level indicates degree of achievement of each behaviour's own desire by value in the range of minimum 0.0 to maximum 1.0. Designed satisfaction level is shown in (4), (5) and (6) and illustrated in Fig. 2 for easy comprehension.

-Satisfaction level of InSafety

$$
\begin{aligned}
& e_{\text {InSafety }}= \\
& \left\{\begin{array}{cc}
\frac{\left(d-|v|_{\max } t_{s}\right)}{r_{\max }-|v|_{\max } t_{s}} & \text { for }|v|_{\max } t_{s} \leq d \leq r_{\max } \\
0 & \text { for }|v|_{\max } t_{s}>d \\
1 & \text { for } r_{\max }<d
\end{array}\right. \\
& \text { where, } e_{\text {InSafety }}: \text { satisfaction level } \\
& d: \text { sensed distance } \\
& |v|_{\max }: \text { maximum possible velocity } \\
& t_{s}: \text { sampling period } \\
& r_{\max }: \text { maximum possible range value } \\
& \text { of ranging sensor }
\end{aligned}
$$

-Satisfaction level of ToGoal

$$
\begin{aligned}
& e_{\text {ToGoal }}= \\
& \left\{\begin{array}{cc}
-\frac{1}{\pi}\left|\theta_{\text {diff }}\right|+1 & \text { for }\left|\theta_{\text {diff }}\right| \leq \pi \\
0 & \text { for }\left|\theta_{\text {diff }}\right|>\pi
\end{array}\right. \\
& \text { where, } e_{\text {ToGoal }} \text { : satisfaction level } \\
& \left|\theta_{\text {diff }}\right| \text { : angle difference } \\
& \text { between the goal direction } \\
& \text { and the robot's current } \\
& \text { heading. }
\end{aligned}
$$

-Satisfaction level of InResolution

$$
\begin{aligned}
& e_{\text {InResolution }}= \\
& \left\{\begin{array}{cc}
\frac{r_{\max }-d}{r_{\max }-|v|_{\max } t_{s}} & \text { for }|v|_{\max } t_{s} \leq d \leq r_{\max } \\
1 & \text { for }|v|_{\max } t_{s}>d \\
0 & \text { for } r_{\max }<d
\end{array}\right. \\
& \text { where, } e_{\text {InResolution }} \text { : satisfaction level } \\
& \text { others are same as (4) }
\end{aligned}
$$



(a) 




(b)

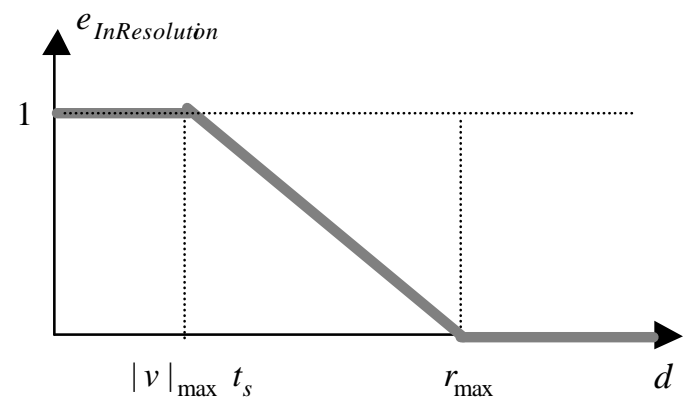

(c)

Fig. 2. Implementation of satisfaction levels for

(a)InSafety, (b)ToGoal, (c)InResolution

Outputs of each behaviours; The behaviour InSafety produces egocentric angular range of inhibition of movement such that

$$
\begin{gathered}
o_{\text {InSafety }}=\left\{\left[\phi_{1 s}, \phi_{1 e}\right],\left[\phi_{2 s}, \phi_{2 e}\right], \cdots\right\} \\
\text { where, } o_{\text {InSafety }} \text { : } \\
{\left[\phi_{s}, \phi_{e}\right]: \text { angultar of InSafety }} \\
\phi_{s} \text { to } \phi_{e} \text { whose corresponding } \\
\text { sonar detect objects. }
\end{gathered}
$$

ToGoal produces egocentric direction for goal such that

$$
\begin{aligned}
& o_{\text {ToGoal }}=\arg (\mathbf{g}-\mathbf{c}) \\
& \quad \text { where, } o_{\text {ToGoal }} \text { :outpur of ToGoal }
\end{aligned}
$$

g:position vector of goal point c:current position vector of the robot $\arg ():$ usual operator which

represents angle of vector.

InResolution produces magnitude of velocity such that

$$
\begin{aligned}
& o_{\text {InResolution }}=\left(1-e_{\text {InResolution }}\right)|v|_{\max } \\
& \text { where, } o_{\text {InResolution }} \text { :output of InResolution }
\end{aligned}
$$

And the velocity command is decided by (9) finally.

\subsection{Behaviour coordination: implementation of navigation}

Real behaviour coordinations occur only inside of each behaviour sectors while behaviour coordinations among different sectors are not necessary. But they only have to be executed in parallel.

Behaviours in a same behaviour sector are coordinated by following scheme. Referring to (2) and Fig. 1, let the name of each behaviour be denoted as behaviour itself and its satisfaction level at the same time for simplicity. Then coordination, which includes step for evaluation of satisfaction in order to explain overall step, is proposed for the sector $i$ as follows:

1.Evaluate satisfaction levels of each homeostatic behaviours.

2.Using the value, select most unsatisfied homeostatic behaviour. e.g. choose homeostatic behaviour candidate $b_{i H}^{*}$ such that

$$
b_{i H}^{*}=\min _{p}\left(b_{i H p}\right), b_{i H p} \in \mathbf{B}_{i H}
$$

3.Change(regulate) the 'internal' states of the robot itself according to $b_{i H}^{*}$ selected at step 2 .

4.Evaluate satisfaction levels of mission behaviours.

5.Select most unsatisfied mission behaviour $b_{i M}^{*}$. e.g.

$$
b_{i M}^{*}=\min _{q}\left(b_{i M q}\right), b_{i M q} \in \mathbf{B}_{i M}
$$

6. Execute behaviour $b_{i M}^{*}$ as final resultant action of sector $i$ under the regulated state of the robot.

In above algorithm, step 1 trough 3 are for checking the homeostasis and others are for choosing mission. Let's see more detail of step 3, the core of this algorithm. In step 3, changing or regulating (term originated from it's original definition in biology) states of action means that the robot refits its parameters related to action such as strength of action, direction of action, etc. to the current environmental states. In (3) or Fig. 1, the behaviour InSafety is the only one homeostatic behaviour in sector 1 and hence it is the most unsatisfied homeostatic behaviour. When the robot moves from free space into front of obstacle, then InSafety changes robot's available moving direction from whole 360 degree into the range excluding the portion covered by obstacle. By this 'homeostatic regulation process', the robot's physical body state itself 
becomes unmovable into obstacle-covered range virtually. This is illustrated in Fig 3. In Fig. 3, the behaviour ToGoal may forces the robot move direction $\leftarrow$ but, by homeostatic regulation, robot already has become non-movable to $\leftarrow$. Hence output of ToGoal in our scheme should reside in movable range and, at the same time, it is better for the output to be as closer to the goal direction as possible. So output of ToGoal is forced change into the direction $\uparrow$. By this scheme, robot can access to goal through short path as well as avoid obstacles. Short path is another merit over other behaviour-based path planning scheme in which improper coordination of velocity components may lead to unnecessarily long path to avoid obstacles.



Fig. 3. Navigation with homeostatic regulation

On the other hand, velocity is controlled independently in behaviour sector 2 regardless of sector 1's output e.g. direction. The behaviour InResolution in sector 2 modulates robot's velocity to fit to current complexity of the environment in such a way that fine control e.g. low velocity when obstacles are nearby while coarse control e.g. fast velocity when obstacles are far away. This may be a remarkable merit since without any additional different kind of efforts, velocity control is accomplished using same consistent behaviour coordination method used path planning and it reflects environmental complexity. By performing these selections of direction in behaviour sector 1 and velocity control in sector 2 at every sampling time, the robot can move reactively for goal direction while avoiding obstacles.

\section{SIMULATIONS AND EXPERIMENTS}

The proposed reactive navigation scheme was simulated graphically and then experiments were performed. Navigation environment was composed of randomly distributed obstacles in a room and the robot(MagellanPro ${ }^{\mathrm{TM}}$ (iRobot corp. 2000)) has cylinder-shape body with 16 equally distributed ultra sonic sensors around it. Fig. 4 shows this navigation environment with a snapshot of experiment. In order to obtain goal direction and to perceive the goal position, odometry system made by optical encoder is used for the time being. It will be replaced by a simple beacon system. But the odometry information is used only for determining the goal direction and other global information such as position coordinates are not used in any way. As the result, successful navigation mission was accomplished as shown in Fig. 5. Here are concrete results obtained;

1.Successfull arrival to goal point.

2.Rare collision cases occurred.

3.Effective short path planned.

4. Velocity control.

5.Cyclic behaviors were resolved to acceptable degree $^{2}$.



Fig. 4. Experiment of reactive navigation

In these results, the second result is the main objective of the homeostatic behaviour coordination scheme since homeostatic behaviour is designed mainly for maintenance of critical and vital status of the robot itself. The third result is as expected in explanation of Fig. 3 where the behaviour ToGoal always maintain tension to the goal even while avoiding obstacles so that the robot does not travel through too far roundabout way. The fourth can be checked with variety of moving distance between each control time step. As far as cyclic behaviour is concerned, although this scheme could not propose perfect solution, as mentioned in remark, acceptable performance about cyclic behaviour was obtained.

${ }^{2}$ Cyclic behaviours occur mainly at box canyon shape terrain and it can be easily shown that complete solution does not 
exist only with reactive navigation scheme. Hence, objective of this research excluded full solution for cyclic behaviours.

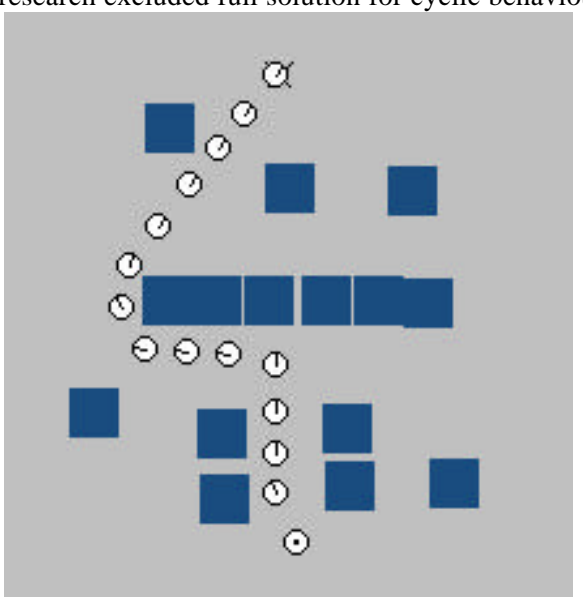

Fig. 5. An accomplishment of navigation mission

Concretely, as shown in Fig. 6, the terrains which are inclined to trap the robot with conventional potential field method were overcame without another endeavour such as gain scheduling, noise addition and memory usage(Arkin, 1987). The behaviour InSafety is a kind of 'free space method' so that (a) and (b) are naturally resolved and turning strategy in only one direction(left or right) when locked by InSafety resolve the long and narrow corridor problem. Although our scheme shown almost perfect performance, several cases of collision and missing goal were obtained. These failures are seemed to authors not due to the proposed algorithm but due to ultra sonic sensor's failure and the incorrect goal direction from odometry.

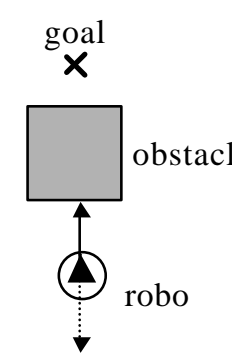

(a)



(c)

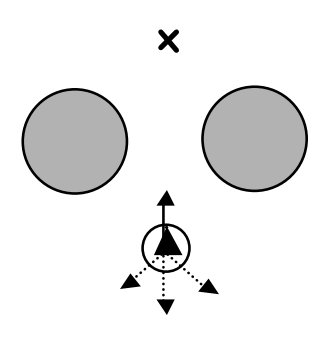

(b)

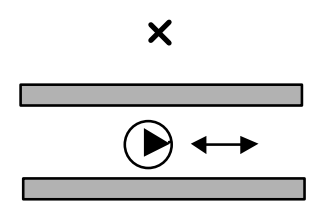

(d)
Fig. 6. Some traps in reactive navigation excluding box-canyon (a)deadlock by single obstacle (b)deadlock by multiple obstacles (c)cyclic moving in front of long obstacle (d)cyclic moving in long tunnel.

\section{CONCLUSIONS}

In this research, a new approach of 'homeostatic' behaviour-based robot control scheme was proposed for reactive navigation and its validities were shown by simulations and experiments. Although the scheme was applied to somewhat simple mission of navigation in this paper, it is a general behaviourbased robot control scheme for any applications which need robot's some degree of intelligence. The proposed homeostatic scheme is believed to be simple, consistent and effective compared to conventional behaviour coordination schemes. Especially, it has merit of guaranteeing the robot not to fall into critical failures. This is more desirable when to apply robots to more complicated missions and environments.

\section{REFERENCES}

Arkin, R.C. (1987). Towards cosmopolitan robots: Intelligent navigation in extended man-made environments, pp143-177, Univ. of Massachusetts, Massachusetts.

Arkin, R.C. (1998). Behavior-based robotics, The MIT Press, Cambridge.

Brooks, R.A. (1986). A robust layered control system for a mobile robot. In: IEEE J. Robotics and Automation, Vol. RA-2, No. 1, pp. 14-23.

iRobot corp. (2000). Magellan Pro User's Guide. (2000), iRobot corp., MA.

Koren, Y. and Borenstein, J. (1991). Potential field methods and their inherent limitations for mobile robot navigation. In:Proc. IEEE Int. Conf. Robotics and Automation, 1991, Sacramento, CA, pp. 1398-1404

Maes, P. (1989). How to Do the Right Thing. In: Connection Science Journal, Vol. 1, No. 3, pp. 291-323

Mukerjee, A., and Mali, A.D. (1999) Reactive robots and amnesics: A comparative study in memoryless behaviour. In: IEEE Trans. Systems, Man, and Cybernetics, Part C: Applications and Reviews, Vol. 292, pp. $216-226$

Saffiotti, A, Konolige, K. and Ruspini, E. (1995). A multivalued logic approach to integrating planning and control. In: Artificial Intelligence, 76, pp 481-526

Xu, W. L. and Tso, S. K. (1999). Sensor-Based Fuzzy Reactive Navigation of a Mobile Robot Through Local Target Switching. In: IEEE Trans. Systems, Man, and Cybernetics, Part C: Applications and Reviews, Vol: 29, NO.3, pp.451-459. 